\title{
New Wideband Transition From Microstrip Line to Substrate Integrated Waveguide
}

\author{
Zamzam Kordiboroujeni, Student Member, IEEE, and Jens Bornemann, Fellow, IEEE
}

\begin{abstract}
A new wideband transition from microstrip line to substrate integrated waveguide (SIW) is introduced. Unlike most transitions that show reduced return loss over significant parts of a regular waveguide band, the presented configuration achieves return losses better than $30 \mathrm{~dB}$ in standard waveguide frequency bands from $X$ to $E$. The new aspect of this transition is the addition of two vias to the widely used microstrip taper transition. Moreover, the influence of the substrate height is demonstrated. The results in each frequency band are compared with the data for the regular microstrip taper alone. A design formula for the placement of the vias and taper dimensions is presented and demonstrated to provide excellent results. The structures are simulated and optimized with CST Microwave Studio. Measurements performed on a Ku-band back-to-back prototype transition demonstrate a minimum return loss of $26.05 \mathrm{~dB}$ and maximum insertion loss of 0.821 dB over the entire Ku-band, thus validating the design approach.
\end{abstract}

Index Terms-Microstrip, substrate integrated waveguide (SIW), taper, transition, wideband.

\section{INTRODUCTION}

$\mathbf{S}$ UBSTRATE integrated waveguide (SIW) technology has made it feasible to design low loss and low interference planar microwave structures. Transitions between SIW and other planar topologies like microstrip and coplanar waveguide (CPW) are needed in order to provide means to excite and measure these structures. More importantly, low-reflection transitions to microstrip are required to integrate and combine SIW circuits with active components such as amplifiers, e.g., [1]. In such applications, it is vital to provide low-reflection transitions so that the component design is independent of the influences of the transitions.

The first interconnect introduced is the microstrip taper [2], and it is still the most widely used type of microstrip-to-SIW transition in single-layered circuits. In [3], the design formula for this type of transition is presented and it is stated that it is generally possible to obtain a return loss $(\mathrm{RL})$ better than $20 \mathrm{~dB}$ over the full waveguide bandwidth ( 40\%) [3]. A microstrip-to-SIW transition with bandwidth of about $24 \%$

Manuscript received March 07, 2014; revised June 08, 2014, September 22, 2014, and October 23, 2014; accepted October 26, 2014. Date of publication November 20, 2014; date of current version December 02, 2014. This work was supported by the National Science and Engineering Research Council of Canada (NSERC).

The authors are with the Department of Electrical and Computer Engineering, University of Victoria, Victoria, BC V8W 3P6, Canada (e-mail: zkordi@ece. uvic.ca; j.bornemann@ieee.org).

Color versions of one or more of the figures in this paper are available online at http://ieeexplore.ieee.org.

Digital Object Identifier 10.1109/TMTT.2014.2365794 and with return loss about $15 \mathrm{~dB}$ for a back-to-back transition in $\mathrm{Ku}$-band is presented in [4]. Another narrowband microstrip-to-SIW transition at $60 \mathrm{GHz}$ in low-temperature cofired ceramic (LTCC) technology is presented in [5]. A return loss of about $15 \mathrm{~dB}$ in the $58-64 \mathrm{GHz}$ range (about $10 \%$ bandwidth) is reported. In [6], a microstrip-to-SIW transition within a multilayered substrate is introduced. With this transition, a bandwidth of $14.5 \%(23.2-27.1 \mathrm{GHz})$ at $15 \mathrm{~dB}$ return loss is obtained. A single-layer dc-coupled microstrip-to-SIW transition using an interdigital configuration is reported in [7]. A return loss about $15 \mathrm{~dB}$ is achieved within a $25 \%$ bandwidth centered at $12.5 \mathrm{GHz}$. Also in 2007, another microstrip-to-SIW transition on LTCC substrate is presented [8]. The return loss for a single transition is reported as $15 \mathrm{~dB}$ within a $30 \%$ bandwidth. A microstrip-to-SIW transition based on an exponential instead of a linear taper is presented in [9]. The return loss is about $20 \mathrm{~dB}$ over a $15 \%$ bandwidth at $18 \mathrm{GHz}$. A transition between SIW and differential microstrip line in multilayered substrate is presented in [10]. Over a $10-\mathrm{GHz}$ bandwidth at $35 \mathrm{GHz}(28 \%)$ a return loss of $10 \mathrm{~dB}$ is achieved. In [11], different types of microstrip tapers in microstrip-to-SIW transitions are investigated and a new design approach based on electromagnetic (EM) simulation is presented. Although the resulting transitions yield return losses better than $30 \mathrm{~dB}$, the structures are very narrow band $(5.5 \%$ at $11 \mathrm{GHz})$. The parallel half-mode SIW (HMSIW) is suggested as transition between microstrip line and SIW structure [12]. This transition relies on the suppression of the dominant higher order TE20 mode, and hence has enhanced bandwidth compared to the conventional microstrip taper. It is stated that the proposed transition has a return loss better than $25 \mathrm{~dB}$ for $1.25 f_{c}-1.9 f_{c}$ with $f_{c}=8.6 \mathrm{GHz}$ [12]. And finally, another narrow band microstrip-to-SIW transition is presented in [13]. According to [13] and for relatively thick substrates, when the characteristic impedance of the SIW is greater than that of the microstrip, the presented transition has better performance compared to the regular microstrip taper. However, except for one case which has a return loss of about $20 \mathrm{~dB}$ between 15 and $40 \mathrm{GHz}$, other presented examples in [13] are narrowband.

In this paper, a new wideband microstrip-to-SIW transition is introduced. It features two vias, which have the same diameter as the SIW vias and are placed symmetrically at both sides of the microstrip taper. This transition provides return losses better than $30 \mathrm{~dB}$ over the entire frequency ranges of standard waveguide bands from $\mathrm{X}$ to $\mathrm{E}(8.2$ to $90 \mathrm{GHz})$ and thus presents a significant improvement over available microstrip-to-SIW transitions. 


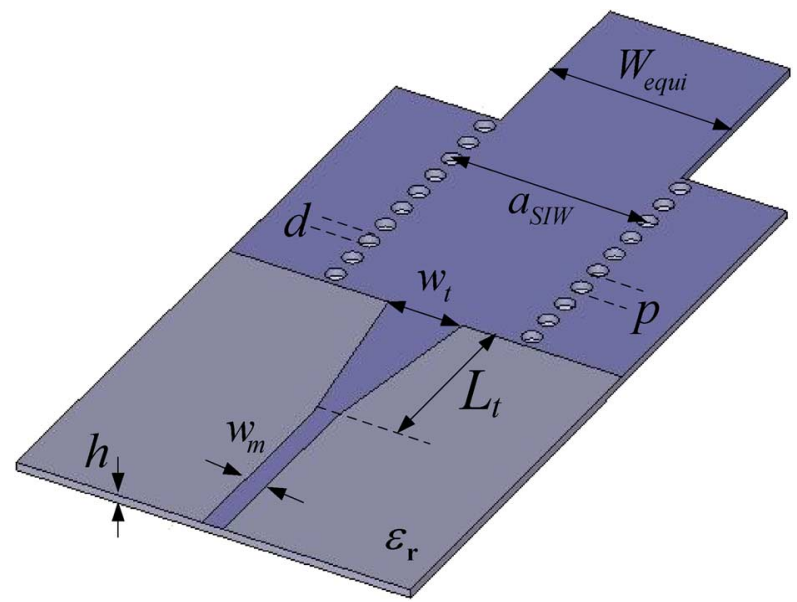

Fig. 1. Structural parameters of a single microstrip taper transition between a microstrip line and an SIW.

In Section II, details of the proposed transition and simulated data based on the optimization for a single transition in different frequency bands are presented. In each frequency band, the presented transition is compared with the regular microstrip taper, and significant improvement in return loss is achieved. In Section III, design equations for designing such transitions are proposed, and transitions designed with these formulas in two new frequency bands are presented. Results are compared with optimized data from EM software which confirm the robustness of the presented design formula. Measurement data for a Ku-band back-to-back transition are presented in Section IV and are in good agreement with simulated data. Section V concludes and provides a summary of the presented results.

\section{TRANSITION TOPOLOGY}

The most common type of microstrip-to-SIW transition in single-layered circuits is the microstrip taper. It not only provides acceptable return loss, but is also wideband and operates over a full waveguide bandwidth [3]. In this section, we present our new transition which improves the return loss significantly compared to the regular microstrip taper. The new transition, which consists of a microstrip taper plus two added vias, proves to be the most wideband microstrip-SIW transition available with minimum return loss.

Fig. 1 presents a single microstrip taper transition from microstrip line to SIW. The other port is terminated with a regular waveguide port.

In this figure, $a_{\mathrm{SIW}}$ is the SIW width, $d$ is the diameter of the vias, $p$ is the via pitch, $w_{m}$ is the width of the microstrip line, $W_{\text {equi }}$ is the width of the waveguide port, $w_{t}$ is the taper width, $L_{t}$ is the taper length, $h$ is the substrate height, and $\varepsilon_{r}$ is the relative permittivity of the substrate.

For the design of an SIW structure, the cutoff frequency $f_{c}$ of the dominant mode $T E_{10}$, substrate permittivity $\varepsilon_{r}$ and $d / p$ ratio (which should be in the practical range of $d / p$ ratios, i.e., $0.5<d / p<0.8$ [14]) are specified. The via pitch is usually chosen such that at least ten vias per guided wavelength are obtained at center frequency. The substrate thickness $h$ is selected based on availability from manufacturers. The cutoff frequencies for the standard waveguide operating frequency bands are

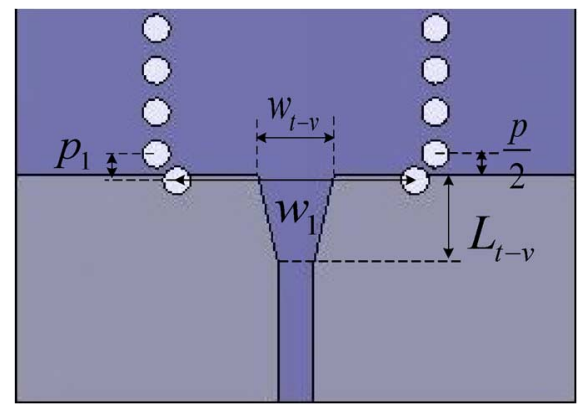

Fig. 2. Structural parameters of the new taper-via transition between microstrip line and SIW.

presented in [15]. Then the SIW width $a_{\mathrm{SIW}}$ is immediately obtained from [16] once the effective width $W_{\text {equi }}$ is calculated from (1), where $\mathrm{c}$ is the speed of light:

$$
W_{\text {equi }}=\frac{c}{2 f_{c} \sqrt{\varepsilon_{r}}} .
$$

In order to excite and integrate an SIW structure with a microstrip port, the first step is to choose $w_{m}$ so that the characteristic impedance of the microstrip line $Z_{0}^{M S}$ becomes $50 \Omega$ at the center frequency of the desired frequency band. Also, it should be noted that depending on the chosen $h$, the characteristic impedance of the SIW structure $Z_{0}^{\text {SIW }}$ differs from $50 \Omega$ and, therefore, different tapering topologies between microstrip line and SIW structure appear. If $h$ is such that $Z_{0}^{\mathrm{SIW}}=$ $Z_{0}^{M S}=50 \Omega$, then no taper transition is needed between the microstrip line and the SIW. This value of $h$ can be found by deploying full-wave software optimizers. In the optimizer, we set $w_{m}=w_{t}$, and try to find $h$ and $w_{m}$ such that $\left|S_{11}\right|$ is minimum for $Z_{0}^{M S}=50 \Omega$ at the center frequency. Such $h_{n t}$ (no-taper $h$ ) values are listed in Table I for all investigated frequency bands in this paper. However, if $h$ is smaller or greater than $h_{n t}$, $Z_{0}^{\mathrm{SIW}}$ is smaller or greater than $50 \Omega$, and we will have taper-out $\left(w_{m}<w_{t}\right)$ or taper-in $\left(w_{m}>w_{t}\right)$ transitions, respectively. In this study, $h$ has been chosen so that we have taper-out taper topologies $\left(h<h_{n t}\right)$, as they are more common in SIW designs. Therefore, the $h_{n t}$ values specified in Table I serve as the maximum substrate height for which the design guidelines presented in this paper apply. Note that the respective exact values of $h_{n t}$ are not regularly available from substrate suppliers.

The new microstrip-to-SIW transition, termed taper-via transition, is presented in Fig. 2. This transition adds two vias to the conventional microstrip taper. The inserted vias are placed symmetrically at both sides of the taper and have the same dimensions as the SIW vias, so there is no need to use a different drill size in the fabrication process.

In Fig. 2, $p_{1}$ is the distance between the inserted via and the first via in the side wall of the SIW, and $w_{1}$ is the distance between the inserted vias. $L_{t-v}$ and $w_{t-v}$ are the length and width of the microstrip taper, respectively.

The advantage of the new configuration compared to the microstrip taper alone is that the field is more confined in lateral direction, and thus a better match from the microstrip to SIW is provided. The variations of the normalized input impedance, 
TABLE I

Structural Parameters of the TAPER Transition and TAPER-Via Transition Between Microstrip LINE AND SIW AT DIFFERENT FREQUENCY BANDS

\begin{tabular}{|c|c|c|c|c|c|c|}
\hline $\begin{array}{l}\text { Frequency band } \\
\text { Parameter }\end{array}$ & $\begin{array}{c}\text { X-band } \\
8.2-12.4 \mathrm{GHz}\end{array}$ & $\begin{array}{c}\text { Ku-band } \\
12.4-18 \mathrm{GHz}\end{array}$ & $\begin{array}{c}\text { K-band } \\
18-26.5 \mathrm{GHz}\end{array}$ & $\begin{array}{c}\text { Ka-band } \\
26.5-40 \mathrm{GHz}\end{array}$ & $\begin{array}{c}\text { U-band } \\
40-60 \mathrm{GHz}\end{array}$ & $\begin{array}{c}\text { E-band } \\
60-90 \mathrm{GHz}\end{array}$ \\
\hline$f_{c}\left(\mathrm{GHz}_{z}\right)$ & 6.557 & 9.488 & 14.051 & 21.077 & 31.391 & 48.373 \\
\hline$p(m m)$ & 2.2 & 1.5 & 1 & 0.7 & 0.45 & 0.3 \\
\hline$d(m m)$ & 1.43 & 0.975 & 0.65 & 0.455 & 0.2925 & 0.195 \\
\hline$a_{S I W}(m m)$ & 14.4124 & 9.9502 & 6.7126 & 4.4913 & 3.0058 & 1.9545 \\
\hline$h_{n t}(m m)$ & 1.6623 & 1.2233 & 0.7990 & 0.5298 & 0.3616 & 0.2210 \\
\hline$h(m m)$ & 0.762 & 0.508 & 0.381 & 0.254 & 0.127 & 0.127 \\
\hline$w_{m}(m m)$ & 1.9161 & 1.2754 & 0.9520 & 0.6358 & 0.3213 & 0.3165 \\
\hline$w_{t}(m m)$ & 3.8868 & 2.7142 & 1.8881 & 1.2476 & 0.7647 & 0.5739 \\
\hline$L_{t}(m m)$ & 5.2611 & 3.7760 & 2.5375 & 1.5357 & 1.1104 & 0.6667 \\
\hline$R L(d B)$ & 21.46 & 21.20 & 21.97 & 21.56 & 19.47 & 23.28 \\
\hline$w_{t-v}(m m)$ & 4.1697 & 2.7533 & 2.0365 & 1.3252 & 0.7784 & 0.6275 \\
\hline$L_{t-v}(m m)$ & 4.2722 & 3.1088 & 1.8908 & 1.3168 & 0.9630 & 0.5458 \\
\hline$w_{1}(\mathrm{~mm})$ & 12.3678 & 8.4486 & 5.7314 & 3.9182 & 2.5078 & 1.7016 \\
\hline$p_{1}(m m)$ & 1.4791 & 0.9494 & 0.6357 & 0.4387 & 0.2840 & 0.2417 \\
\hline$R L(d B)$ & 31.53 & 35.2 & 32.6 & 31.84 & 32.19 & 29.02 \\
\hline
\end{tabular}

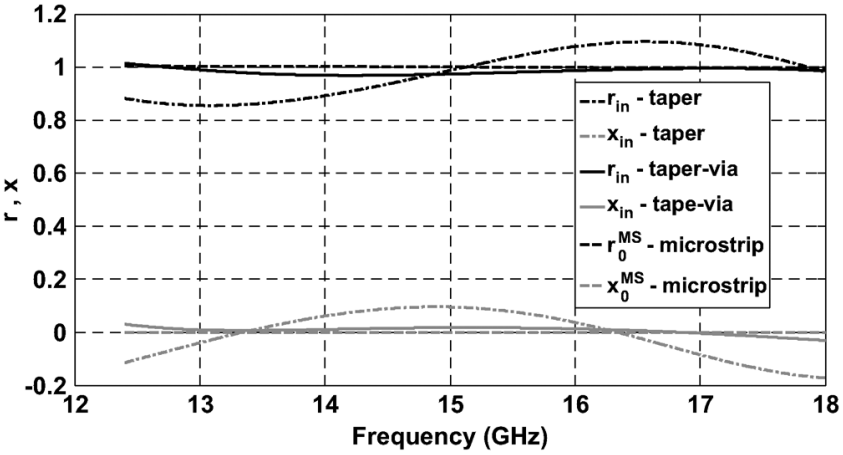

Fig. 3. Comparison between real (black) and imaginary (grey) parts of the input impedance for three different cases: structure with taper transition (dotted-dashed line), structure with taper-via transition (solid line), microstrip line (dashed line).

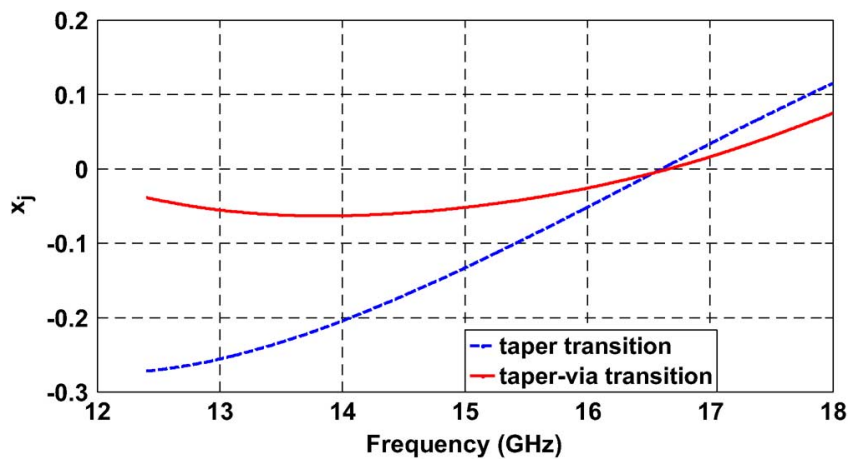

Fig. 4. Normalized reactance comparison in the microstrip-to-SIW junction plane for both taper (dashed line) and taper-via (solid line) transitions.

$z_{i n}=Z_{i n} / Z_{0}^{M S}=r_{i n}+j x_{i n}$, with frequency are plotted in Fig. 3 for both taper and taper-via transitions and are compared with the normalized characteristic impedance of the microstrip line. It is observed that the taper-via transition provides an input impedance closer to $Z_{0}^{M S}$, compared to the taper transition alone, which proves that better matching between the microstrip line and the SIW structure is achieved.

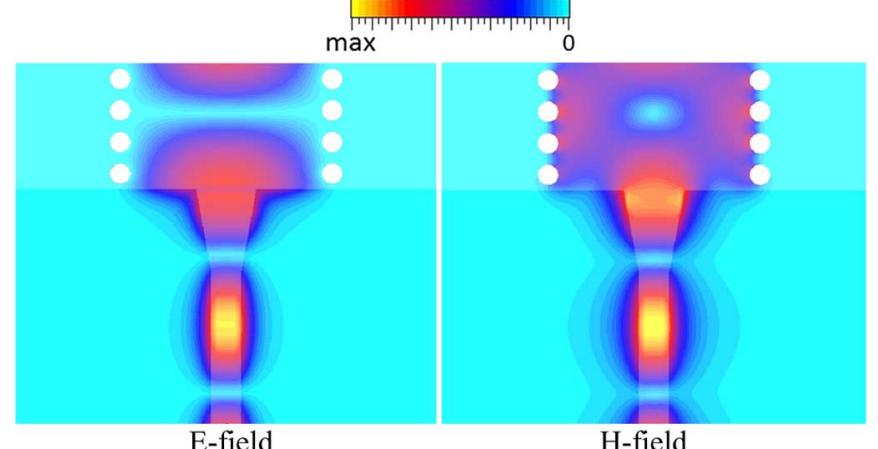

(a)
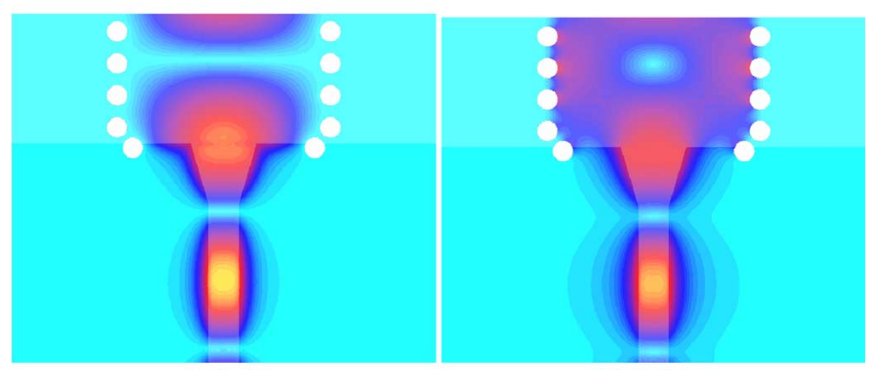

(b)

H-field

Fig. 5. Magnitudes of electric and magnetic field patterns of transitions between a microstrip line and an SIW: (a) conventional taper; (b) new transition.

In order to investigate the nature of this improved performance achieved by the proposed transition, the normalized reactance of the microstrip-to-SIW junction $\left(x_{j}\right)$ is compared for both taper and taper-via transitions. As shown in Fig. 4, the added vias to the taper transition compensate the reactance effect of the microstrip taper-to-SIW transition in the junction plane and provide an overall reactance that is, over the entire bandwidth, smaller than that of the regular transition.

The field patterns in the new transition are also compared to those of the microstrip taper alone and presented in Fig. 5. In all four cases in Fig. 5, the same scaling has been adopted so that the $a_{\mathrm{SIW}}$ and $d$ are exactly the same. Also, the phase of the incident wave has been chosen so that for both transitions, the 


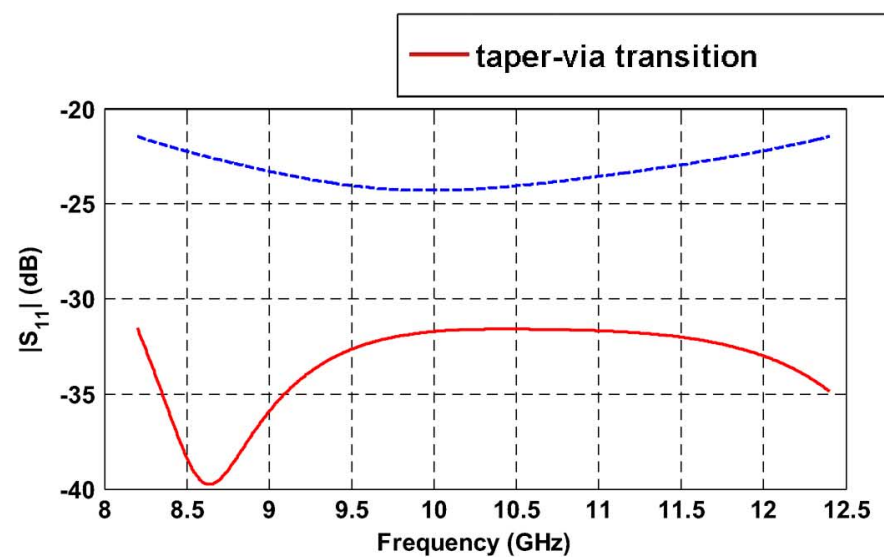

(a)

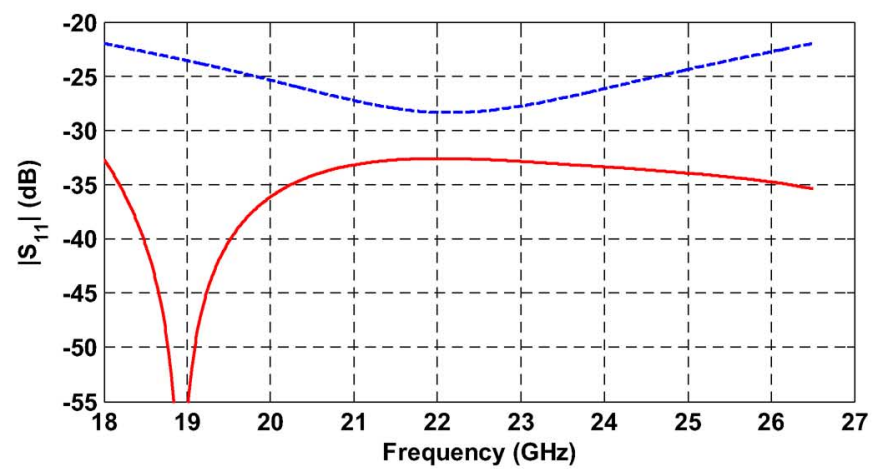

(c)

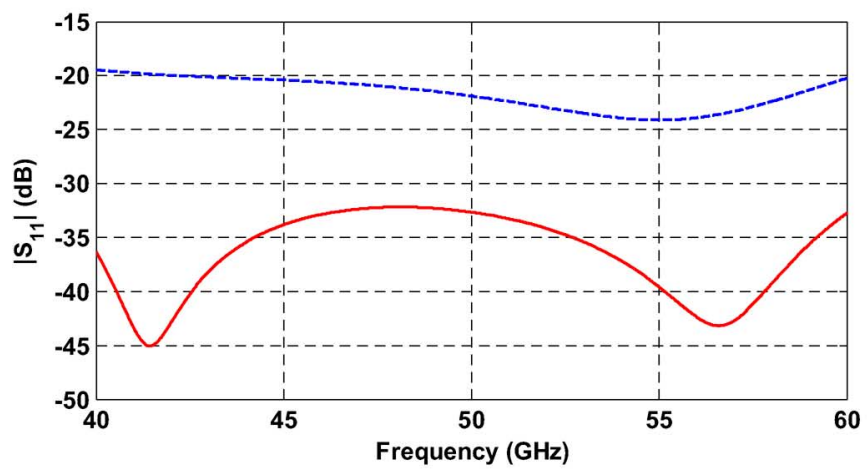

(e)

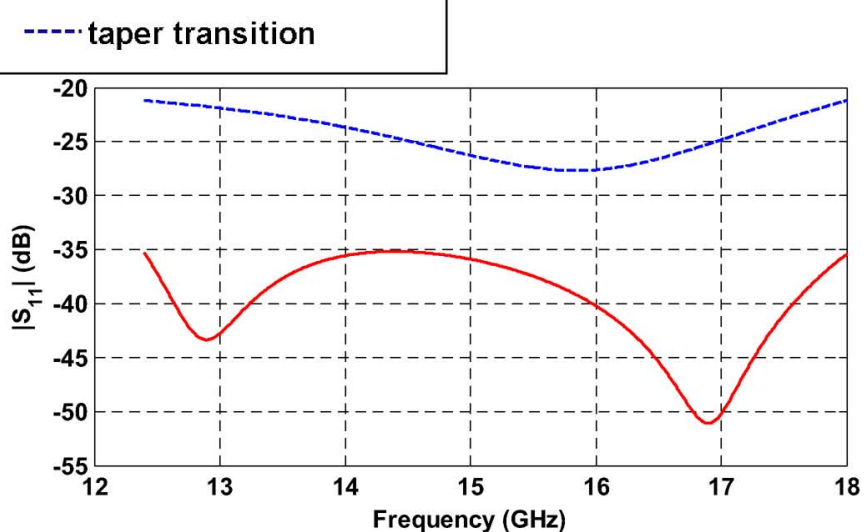

(b)

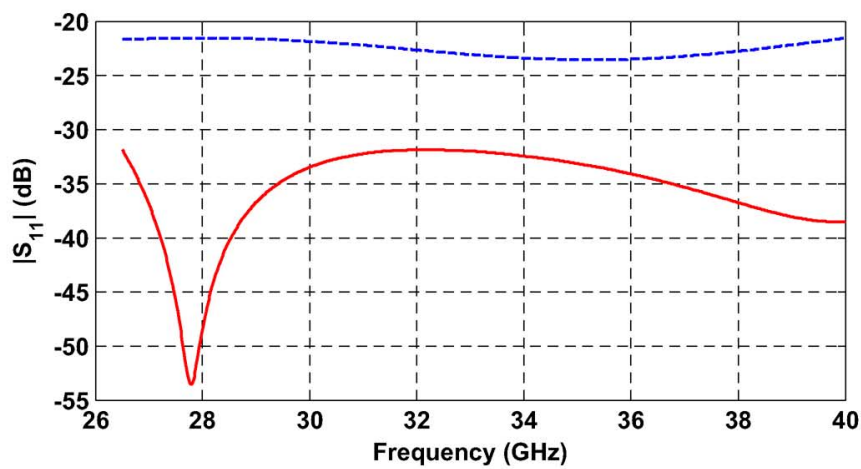

(d)

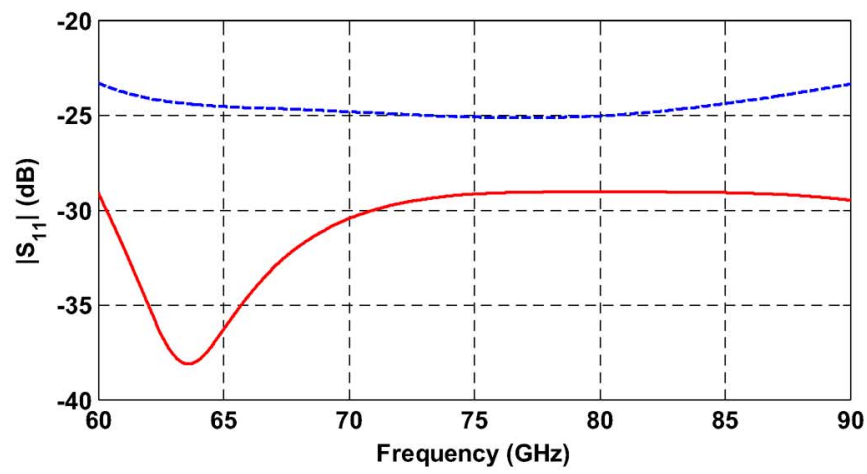

(f)

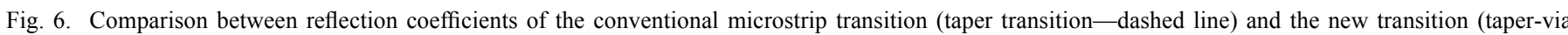

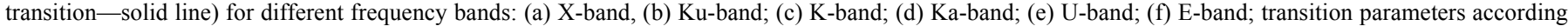
to Table I.

maximum E-field occurs at the beginning of the SIW structure. The H-fields are plotted at the same phase. As it is observed, by confining the field better, the new transition results in better matching between microstrip and SIW, and thus has lower return loss. When the proposed transition is deployed, the electromagnetic field of the microstrip line attaches itself better, e.g., with lower fringing fields, thus reduced lateral field extension, at the SIW interface, to the vias [Fig. 5(b)] compared to the traditional taper [Fig. 5(a)].

Based on the new configuration in Fig. 2, transitions in different microwave frequency bands from 8.2 to $90 \mathrm{GHz}$ have been designed. In all cases, the performance of the new transition is compared with that of the conventional microstrip taper. The substrate material in this investigation is chosen as RT/duroid 6002 with effective permittivity $\varepsilon_{r}=2.94$ for both taper and taper-via transitions. (Note that design formulas introduced in Section III can be applied to any other substrate.) The SIW structure consists of ten rows of vias. Taper-via transition parameters $w_{t-v}, L_{t-v}, p_{1}, w_{1}$ (cf. Fig. 2) are optimized in the frequency-domain solver of CST Microwave Studio in order to maximize the return loss of a single transition over the entire waveguide band. The optimized parameters are presented in Table I.

Optimized parameters of conventional tapers $\left(w_{t}, L_{t}\right)$ are also presented in Table I. It is worth mentioning that the optimized parameters of the conventional tapers differ from values obtained from the formulation presented in [3]. In each frequency band, the substrate height $h$ is chosen so that we have taper-out microstrip-SIW transitions $\left(h<h_{n t}\right)$ as they are much more common than taper-in transitions. For this height 


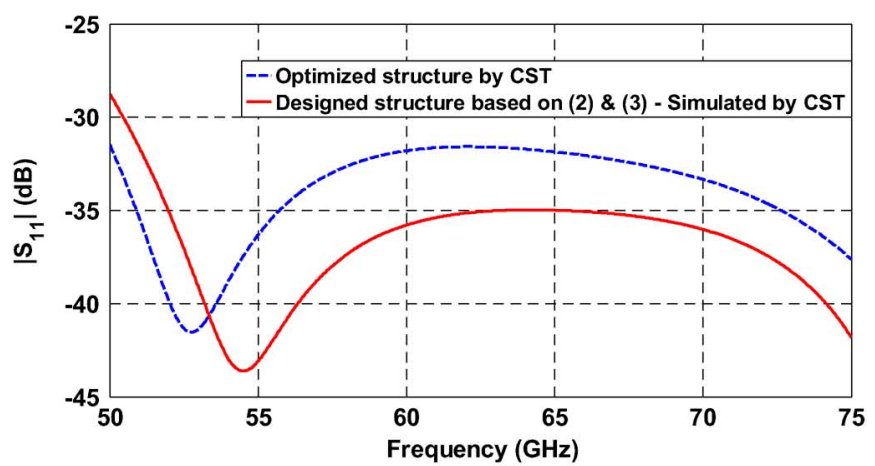

(a)

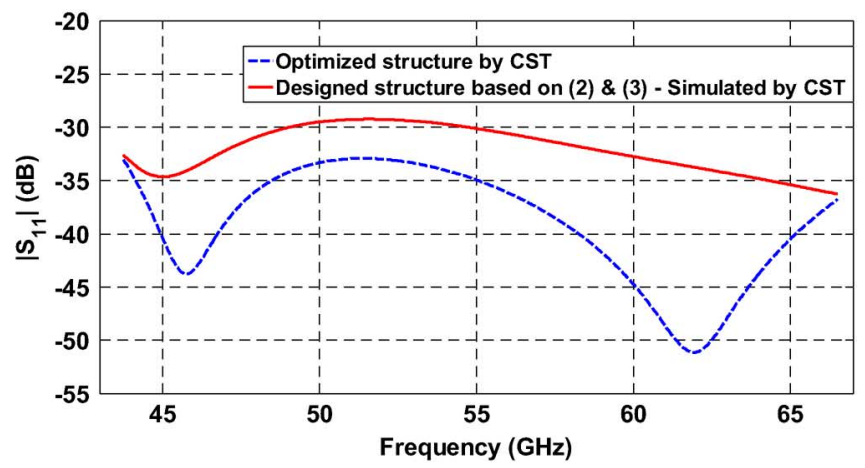

(b)

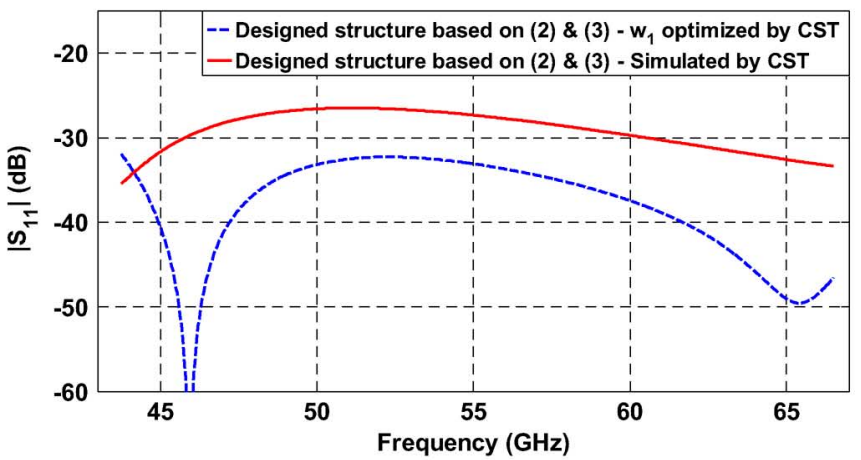

(c)

Fig. 7. Examples showing comparison between the reflection coefficient of the transition optimized in CST (blue - dashed line), and the performance of the transition designed based on (2) and (3) (red - solid line), cf. Table II: (a) Example 1; (b) Example 2; (c) Example 3.

$(h), w_{m}$ is calculated so that we have $Z_{0}^{M S}=50 \Omega$ at the center frequency.

In Fig. 6, the reflection coefficients of the new transitions are compared with those of the conventional microstrip taper in each frequency band. It is observed that the new transitions show return losses better than $30 \mathrm{~dB}$ within each frequency band if the dielectric height is chosen properly (cf. Table I). (Note that a direct scaling process of the new transition to other bands would involve the height of the substrate material and will result in substrate heights that are not readily available from suppliers.) The new transition has significantly improved performance compared to the conventional taper transition. For the E-band, the $5 \mathrm{mil}(0.127 \mathrm{~mm})$ substrate is the only option provided by the manufacturer for maintaining $h<h_{n t}$. The new E-band transition [Fig. 6(f)] still outperforms the conventional taper transition by close to $6 \mathrm{~dB}$ in this case. However, if a
TABLE II

Structural Parameters of the TAPER-Via Transition BetweEN MICROSTRIP LINE AND SIW FOR DIFFERENT EXAMPLES

\begin{tabular}{|c|c|c|c|}
\hline $\begin{array}{r}\text { Frequency } \\
\text { band } \\
\text { Parameter }\end{array}$ & $\begin{array}{c}\text { Ex. 1 } \\
\text { V-band } \\
\text { 50-75 GHz }\end{array}$ & $\begin{array}{c}\text { Ex. } 2 \\
43.75-66.5 \\
\text { GHz }\end{array}$ & $\begin{array}{c}\text { Ex. 3 } \\
43.75-66.5 \\
\text { GHz }\end{array}$ \\
\hline$f_{c}(\mathrm{GHz})$ & 39.875 & 35 & 35 \\
\hline$\varepsilon_{r}$ & 2.94 & 6.15 & 2.2 \\
\hline$p(m m)$ & 0.36 & 0.28 & 0.47 \\
\hline$d(m m)$ & 0.234 & 0.21 & 0.2585 \\
\hline$a_{S I W}(m m)$ & 2.3691 & 1.8947 & 3.0646 \\
\hline$h(m m)$ & 0.127 & 0.127 & 0.127 \\
\hline$w_{m}(m m)$ & 0.3184 & 0.1815 & 0.3894 \\
\hline$L_{t-v}^{\text {design }}(\mathrm{mm})$ & 0.7126 & 0.5980 & 0.9141 \\
\hline$L_{t-v} C S T(m m)$ & 0.7134 & 0.6194 & 0.9141 \\
\hline$w_{t-v}{ }^{\text {design }}(\mathrm{mm})$ & 0.6849 & 0.4747 & 0.8635 \\
\hline$w_{t-v} C S T(\mathrm{~mm})$ & 0.6844 & 0.4782 & 0.8635 \\
\hline$p_{1}^{\text {design }}(\mathrm{mm})$ & 0.2362 & 0.1837 & 0.3084 \\
\hline$p_{1} C S T(\mathrm{~mm})$ & 0.2599 & 0.1854 & 0.3084 \\
\hline$w_{1}^{\text {design }}(\mathrm{mm})$ & 2.0269 & 1.6211 & 2.6221 \\
\hline$w_{1}^{C S T}(\mathrm{~mm})$ & 2.0173 & 1.6146 & 2.5168 \\
\hline
\end{tabular}

thinner substrate is available, the performance improvement of the new transition is more significant compared to the taper alone. The minimum return loss values of all transitions in Fig. 6 are also presented in Table I.

\section{Design Formulation}

Based on the values presented in Table I, we extract formulas for the direct design of the new transition in Fig. 2. After calculation of SIW and microstrip parameters for the desired frequency band, the next step for designing a taper-via transition is to calculate the transition parameters $w_{t-v}, L_{t-v}, p_{1}, w_{1}$ based on the following simple formulations:

$$
\begin{aligned}
L_{t-v} & =0.2368 \lambda_{g-m s} ; w_{t-v}=w_{m}+0.1547 a_{\mathrm{SIW}} \\
p_{1} & =0.6561 p ; w_{1}=0.8556 a_{\mathrm{SIW}}
\end{aligned}
$$

in which $\lambda_{g-m s}$ is the guided wavelength of the microstrip line calculated at the center frequency

$$
\lambda_{g-m s}=\frac{\lambda_{g 0}}{\sqrt{\varepsilon_{\text {reff }}}} .
$$

$\lambda_{g 0}$ is the wavelength in free space, and $\varepsilon_{r e f f}$ is the effective dielectric constant of the microstrip line, both calculated at the center frequency. As it can be seen from (2), $L_{t-v}$ is always close to a quarter of $\lambda_{g-m s}$. Also, the difference between $w_{t-v}$ and $w_{m}$ is approximately about one-eighth of $a_{\text {SIW }}$. The placement of the two added vias is also related to SIW parameters $p$ and $a_{\text {SIW }}$ (cf. Fig. 1). Based on (2) and (3), the normalized root-mean-square errors of $L_{t-v}, w_{t-v}, p_{1}$ and $w_{1}$ to the original data in Table I are 3, 1.2, 3, and $0.6 \%$, respectively.

Based on the proposed formulas, three examples have been investigated. Example 1 [Fig. 7(a)] presents the new taper-via transition in V-band. The $d / p$ ratio and the substrate are chosen as in the previous structures. Examples 2 and 3 present the performances of the two new taper-via transitions at an arbitrary frequency band of between 43.75 and $66.5 \mathrm{GHz}$, where the waveguide cut-off frequency is $35 \mathrm{GHz}$. In Example 2 [Fig. 7(b)], the dielectric is chosen as RT/duroid 6006 with 


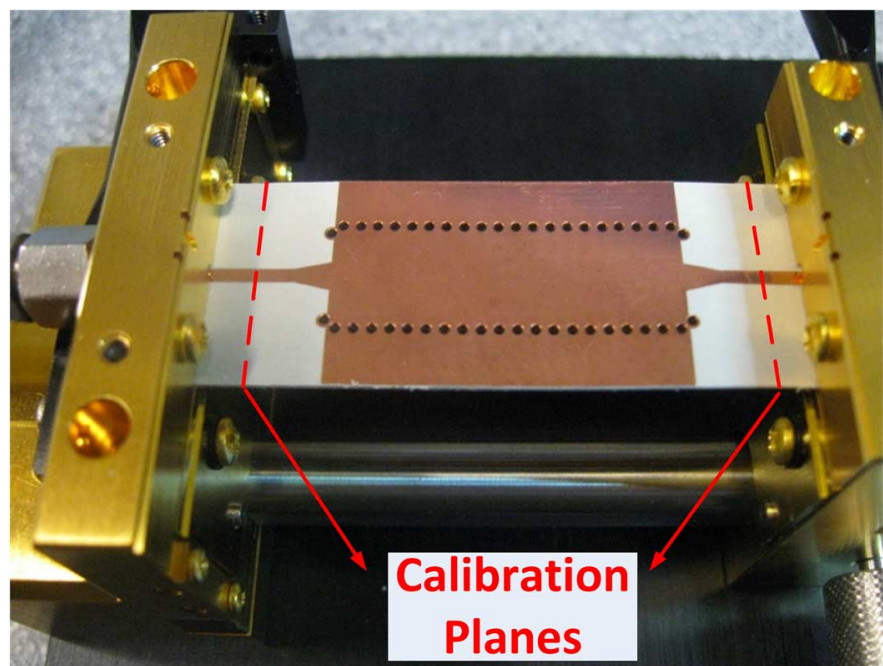

Fig. 8. Back-to-back fabricated taper-via transition at Ku-band and indication of calibration planes.

$\varepsilon_{r}=6.15$ and $d / p=0.75$, and in Example 3 [Fig. 7(c)], the dielectric is chosen as RT/duroid 5580 with $\varepsilon_{r}=2.2$ and $d / p=0.55$. In each case, the performances of the new transitions [designed from (2)-(4)] are compared with those of optimized transitions in CST.

The results are presented in Fig. 7 with structural parameters given in Table II. In this table, the structural parameters of the optimized structure by CST are presented by superscript "CST," while the values obtained from the design formulas are presented by superscript "design." It is observed that the presented design formulas result in transitions that perform close to the optimized performance [Fig. 7(a), (b)]. In some cases, however, the performance of the transition based on the design formulas is not the best possible performance (RL around $27 \mathrm{~dB}$ in Fig. 7(c) - solid line). A few optimization steps towards adjusting just one parameter (here $w_{1}$ ) will bring $\left|\mathrm{S}_{11}\right|$ below $-30 \mathrm{~dB}$ (dashed line in Fig 7(c)). Nevertheless, the examples presented in Fig. 7 validate the simple design formulations presented in (2), (3).

\section{Measurements}

A back-to-back version of the new taper-via transition in Ku-band has been prototyped. Fig. 8 shows the fabricated structure under test. For measurements, an LRL calibration (thru-short-line) is used to deembed the influences of the test fixture and its coaxial connectors. The calibration planes are located in the feeding microstrip lines as shown in Fig. 8.

The back-to-back transition was originally designed and optimized in CST considering dielectric and conductor losses $\left(\tan \delta=0.0012, \sigma_{c}=5.8 \times 10^{7} \mathrm{~S} / \mathrm{m}\right)$. In Fig. 9, the performance of the originally optimized structure (solid lines) is presented. The original via diameter of the Ku-band structure (cf. Table I) was changed from $0.975 \mathrm{~mm}$ to $0.965 \mathrm{~mm}$ due to drill size restrictions. Also, in the manufacturing process a minimum amount of conductor plating was necessary around the top of the left- and right-most vias. The structure is again simulated in CST including the fabrication restrictions, and its

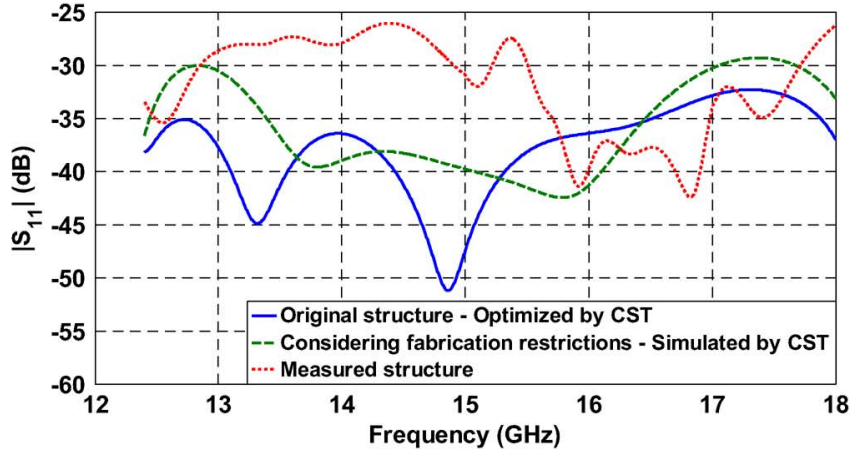

(a)

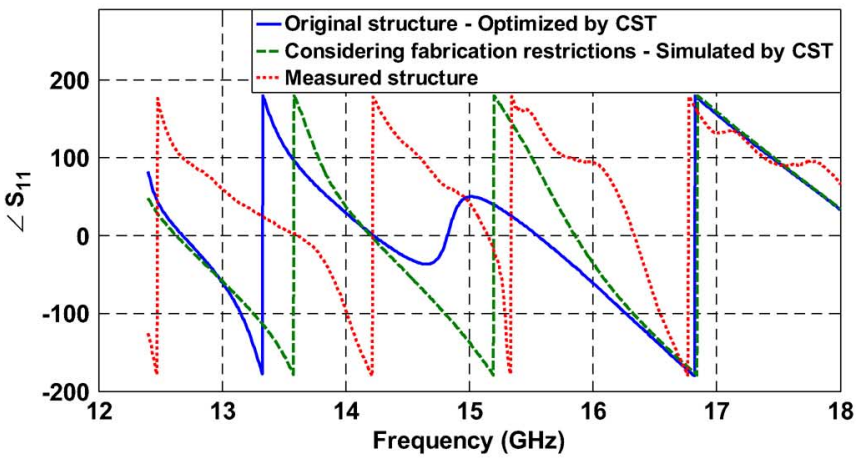

(b)

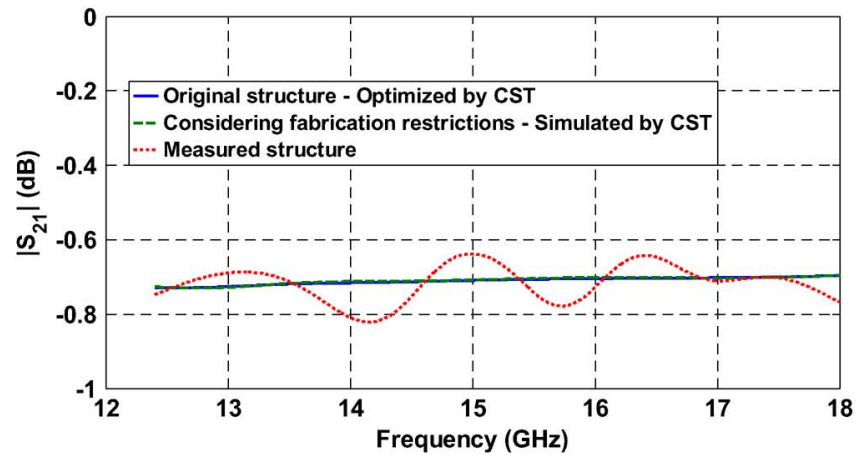

(c)

Fig. 9. Comparison between the S-parameters of the original transition optimized in CST (solid lines), the structure considering manufacturing restrictions, simulated in CST (dashed lines), and the measurement data (dotted lines): (a) reflection coefficient (amplitude); (b) reflection coefficient (phase); (c) transmission coefficient (amplitude).

performance is presented in Fig. 9 as dashed lines for comparison with measured data (dotted lines).

It is worth mentioning that symmetric conductor plating around the outside vias is considered in the simulation, whereas the actual plated metals in the fabricated prototype are asymmetric. This is the main reason for the difference between simulated and measured results. However, the measured return loss (Fig 9(a), dotted line) is better than $26.05 \mathrm{~dB}$ in the entire $\mathrm{Ku}$-band which, to the best of the authors' knowledge, is the lowest measured return loss over a full waveguide band for the microstrip-to-SIW transitions reported in the literature. In addition, the difference between the phases of the structures [Fig. 9(b)] is due to the different added metal plating, as it plays significant roles in the phase response [compare the data for the two simulated structures, solid line and dashed line 
data, in Fig. 9(b)]. Other than that, the phases have similar patterns. The maximum measured insertion loss of the fabricated back-to-back transition is $0.821 \mathrm{~dB}$ [Fig. 9(c)] and is about $0.1 \mathrm{~dB}$ lower than the prediction by CST.

\section{CONCLUSION}

A new wideband transition from microstrip line to SIW is presented. It shows return loss values of about $30 \mathrm{~dB}$ over entire waveguide frequency bands, which is the lowest return loss achieved over wide frequency bands. The performance of the transition is presented in the waveguide frequency bands ranging from 8.2 to $90 \mathrm{GHz}$. The formula introduced for the design of wideband and low-reflection transitions is demonstrated to provide simplicity as well as robustness. The measured return loss for the back-to-back taper-via transition in Ku-band is better than $26.05 \mathrm{~dB}$, which is the lowest measured return loss available over a full waveguide band.

We hope that this work will inspire similar improvements to other transitions between SIW and planar technologies such as CPW [17], grounded CPW [18], coplanar stripline (CPS) and slotline [19].

\section{REFERENCES}

[1] M. Abdolhamidi and M. Shahabadi, "X-Band substrate integrated waveguide amplifier," IEEE Microw. Wireless Compon. Lett., vol. 18, no. 12, pp. 815-817, Dec. 2008.

[2] D. Deslandes and K. Wu, "Integrated microstrip and rectangular waveguide in planar form," IEEE Microw. Wireless Compon. Lett., vol. 11, no. 2, pp. 68-70, Feb. 2001.

[3] D. Deslandes, "Design equations for tapered microstrip-to-substrate integrated waveguide transitions," in IEEE MTT-S Int. Microw. Symp. (IMS) Dig., Anaheim, CA, USA, May 2010, pp. 704-707.

[4] H. Nam, T.-S. Yun, K.-B. Kim, K.-C. Yoon, and J.-C. Lee, "Ku-band transition between microstrip and substrate integrated waveguide (SIW)," in Proc. Asia-Pacific Microw. Conf. (APMC), Suzhou, China, Dec. 2005, pp. 1-4.

[5] T.-H. Yang, C.-F. Chen, T.-Y. Huang, C.-L. Wang, and R.-B. Wu, "A $60 \mathrm{GHz}$ LTCC transition between microstrip line and substrate integrated waveguide," in Proc. Asia-Pacific Microw. Conf. (APMC), Suzhou, China, Dec. 2005, pp. 1-4.

[6] Y. Ding and K. Wu, "Substrate integrated waveguide-to-microstrip transition in multilayer substrate," IEEE Trans. Microw. Theory Tech., vol. 55, no. 12, pp. 2839-2844, Dec. 2007.

[7] M. Abdolhamidi, A. Enayati, M. Shahabadi, and R. Faraji-Dana, "Wideband single-layer DC-decoupled substrate integrated waveguide (SIW) - to-Microstrip transition using an interdigital configuration," in Proc. Asia-Pacific Microw. Conf. (APMC), Bangkok, Thailand, Dec. 2007, pp. 1-4.

[8] C.-K. Yau, T.-Y. Huang, T.-M. Shen, H.-Y. Chien, and R.-B. Wu, "Design of $30 \mathrm{GHz}$ transition between microstrip line and substrate integrated waveguide," in Proc. Asia-Pacific Microw. Conf. (APMC), Bangkok, Thailand, Dec. 2007.

[9] Z. Sotoodeh, B. Biglarbegian, F. H. Kashani, and H. Ameri, "A novel bandpass waveguide filter structure on SIW technology," Progress Electromagn. Res. Lett., vol. 2, pp. 141-148, 2008.

[10] F. Bauer and W. Menzel, "A wideband transition from substrate integrated waveguide to differential microstrip lines in multilayer substrates," in Proc. 40th Eur. Microw. Conf. (EuMC), Paris, France, Sep. 2010, pp. 811-813.

[11] E. Miralles, H. Esteban, C. Bachiller, A. Belenguer, and V. E. Boria, "Improvement for the design equations for tapered microstrip-to-substrate integrated waveguide transitions," in Proc. Int. Conf. Electromagn. Adv. Applicat. (ICEAA), Torino, Italy, Sep. 2011, pp. 652-655.

[12] D.-K. Cho and H.-Y. Lee, "A new broadband microstrip-to-SIW transition using parallel HMSIW," J. Electromagn. Eng. Sci., vol. 12, no. 2, pp. 171-175, Jun. 2012.
[13] E. D. Caballero, A. B. Martinez, H. E. Gonzalez, O. M. Belda, and V. B. Esbert, "A novel transition from microstrip to a substrate integrated waveguide with higher characteristic impedance," in IEEE MTT-S Int. Microw. Symp. (IMS) Dig., Seattle, WA, USA, Jun. 2013, pp. 1-4.

[14] D. Deslandes and K. Wu, "Accurate modeling, wave mechanisms, design considerations of a substrate integrated waveguide," IEEE Trans. Microw. Theory Tech., vol. 54, no. 6, pp. 2516-2526, Jun. 2006.

[15] A. F. Harvey, "Standard waveguides and couplings for microwave equipment," Proc. IEE_Part B: Radio Electron. Eng., vol. 102, no. 4, pp. 493-499, Jul. 1955.

[16] Z. Kordiboroujeni and J. Bornemann, "Designing the width of substrate integrated waveguide structures," IEEE Microw. Wireless Compon. Lett., vol. 23, no. 10, pp. 518-520, Oct. 2013.

[17] F. Taringou, J. Bornemann, K. Wu, and T. Weiland, "Broadband interconnects between coplanar waveguide and substrate integrated waveguide for dense packaging and integration," in IEEE MTT-S Int. Microw. Symp. (IMS) Dig., Tampa, FL, USA, Jun. 2014, pp. 1-3.

[18] X.-P. Chen and K. Wu, "Low-loss ultra-wideband transition between conductor-backed coplanar waveguide and substrate integrated waveguide," in IEEE MTT-S Int. Microw. Symp. (IMS) Dig., Boston, MA, USA, Jun. 2009, pp. 349-352.

[19] F. Taringou, D. Dousset, J. Bornemann, and K. Wu, "Substrate-integrated waveguide transitions to planar transmission-line technologies," in IEEE MTT-S Int. Microw. Symp. (IMS) Dig., Montreal, QC, Canada, Jun. 2012, pp. 1-3.

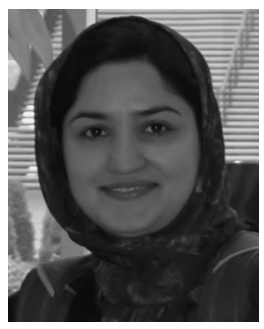

Zamzam Kordiboroujeni received the B.Sc. and M.Sc. degrees in electrical engineering from the Iran University of Science and Technology, Tehran, Iran, in 2005 and 2008, respectively. She is currently working towards the Ph.D. degree in the Department of Electrical and Computer Engineering, University of Victoria, Victoria, BC, Canada.

She is a Research Assistant in the Computer-Aided Design of Microwave Integrated Circuits (CADMIC) research group. Her current research interests include SIW technology and computational electromagnetics.

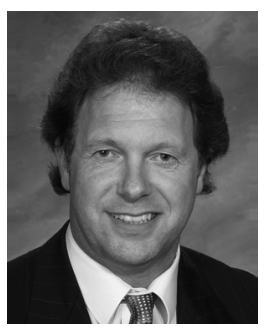

Jens Bornemann (M'87-SM'90-F'02) received the Dipl.-Ing. and the Dr.-Ing. degrees, both in electrical engineering, from the University of Bremen, Bremen, Germany, in 1980 and 1984, respectively.

From 1984 to 1985, he was a Consulting Engineer. In 1985, he joined the University of Bremen, as an Assistant Professor. Since April 1988, he has been with the Department of Electrical and Computer Engineering, University of Victoria, Victoria, BC, Canada, where he became a Professor in 1992. From 1992 to 1995 , he was a Fellow of the British Columbia Advanced Systems Institute. In 1996, he was a Visiting Scientist at Spar Aerospace Limited (now MDA Space), Ste-Anne-de-Bellevue, Québec, Canada, and a Visiting Professor at the Microwave Department, University of Ulm, Germany. From 1997 to 2002, he was a codirector of the Center for Advanced Materials and Related Technology (CAMTEC), University of Victoria. In 2003, he was a Visiting Professor at the Laboratory for Electromagnetic Fields and Microwave Electronics, ETH Zurich, Switzerland. He has coauthored Waveguide Components for Antenna Feed Systems-Theory and Design (Artech House, 1993) and has authored/coauthored more than 300 technical papers. His research activities include RF/wireless/microwave/millimeter-wave components and systems design, and field-theory-based modeling of integrated circuits, feed networks and antennas.

Dr. Bornemann served as an Associate Editor of the IEEE TRANSACTIONS ON MicRowAVE THEORY AND TECHNIQUES from 1999 to 2002 in the area of microwave modeling and CAD. From 2006 to 2008, he was an Associate Editor of the International Journal of Electronics and Communications. From 1999 to 2009 , he served on the Technical Program Committee of the IEEE MTT-S International Microwave Symposium. He is a Registered Professional Engineer in the Province of British Columbia, Canada. He is a Fellow of the Canadian Academy of Engineering (CAE) and serves on the editorial advisory board of the International Journal of Numerical Modelling. 